\title{
Dimension Fractal in Radiological Imagery for Comparison of Data Between Morphologic and Pathological Elements
}

\author{
Ernesto Borges Batista ${ }^{1}$, Luis Alberto Escalona Fernandez ${ }^{2,}$, , Kirelis Napoles Dominguez, \\ Yamila Ochoa Sarmiento ${ }^{1}$, Claudia del Carmen Pupo Marrero ${ }^{1}$ \\ ${ }^{1}$ Department of Basic Biomedical Sciences, Holguin University of Medical Sciences, Holguin, Cuba \\ ${ }^{2}$ Department of Medical Information Technology, Faculty of Medical Sciences, Holguin University of Medical Sciences, Holguin, Cuba
}

Email address:

luisalbert@infomed.sld.cu (L. A. E. Fernandez)

${ }^{*}$ Corresponding author

\section{To cite this article:}

Ernesto Borges Batista, Luis Alberto Escalona Fernandez, Kirelis Napoles Dominguez, Yamila Ochoa Sarmiento, Claudia del Carmen Pupo Marrero. Dimension Fractal in Radiological Imagery for Comparison of Data Between Morphologic and Pathological Elements. Applied and Computational Mathematics. Vol. 10, No. 2, 2021, pp. 40-45. doi: 10.11648/j.acm.20211002.12

Received: December 11, 2020; Accepted: January 7, 2021; Published: June 16, 2021

\begin{abstract}
Aims: Fractal for comparison of radiological imagery between morphologic and pathological elements confirms the behavior of the experimental information through dimension itself. The irregularity of the human body is its own characteristic. However, it has traditionally been measured with Euclidean metrics, by approximating its shapes to regular lines, areas and volumes. In response to this impossibility of making reliable measurements of this class of objects, fractal geometry is developed, which allows to adequately characterize the irregular shape of the human body. Method: they use the theoretic methods: Analysis synthesis, induction deduction and abstraction concretion. Processes of understanding, explanation and interpretation. Methods, procedures and mathematical algorithms, as well as information-technology professional programs are applicable. Come true quest of information about the application of dimension fractal in the diagnostic one belonging to diseases, based in radiological imagery. The diagnostic method fractal consists in the calculation of dimension for three cellular objects defined as: the nucleus, the cytoplasm without a nucleus and the entire cell. Results: Methods and procedures to ratify diseases, where the different authors yield a mathematical model, propose which themselves fractal for the comparison of histological and pathological elements confirms the behavior of the experimental data represented in radiological imagery, by means of dimension. About fractal geometry, the fractal dimension is obtained, which is a numerical measure that represents the degree of irregularity of an object. However, it has traditionally been measured with Euclidean metrics, by approximating its shapes to regular lines, areas and volumes. In response to this impossibility of making reliable measurements of this class of objects, fractal geometry is developed, which allows to adequately characterize the irregular shape of the human body. Conclusions: A methodology of work based in radiological imagery by comparison of histological and pathological elements to determine different diseases in patients becomes established.
\end{abstract}

Keywords: Experimental Data, Mathematical Model, Medical Applications

\section{Introduction}

What do clouds, mountain ranges, coasts, trees, rivers and some natural phenomena have in common with our biology, say Nervous, Cardiovascular, Neurological System plus some pathological processes that affect us as a species? All have in common the presence of patterns approximated to Fractals and their Mathematics that develops them. [1, 2, 3]

In order to understand their use in medical sciences, we must have even a basic notion of their properties or concepts that make up this branch of mathematics, so a fractal; It is a geometric object whose basic structure, fragmented or apparently irregular, is repeated at different scales, this is one of its most important properties, the self-similarity or selfsimilarity that its structure has on a smaller scale of magnitude, starting from a mother figure or seed, to infinity. [2, 4, 5]

This would be the ideal fractal, the classic mathematical equation that is repeated or iterated infinity of times (Figure 1) resulting in that its fractal metric dimension is a rational 
number greater than its topological dimension or its Hausdorff - Besicovitch dimension is strictly greater than its topological dimension, which in non-mathematical language have an infinite perimeter in a finite area. [2]

That is why we say that, with respect to our biology organized by system and the natural world that surrounds us, there are well-organized structural patterns approximated to fractal forms, since by simple physics, the structural form of matter visually changes to a lesser scale and loses its selfsimilarity, which can only be observed at macroscopic scales (Figure 2). [1, 2]

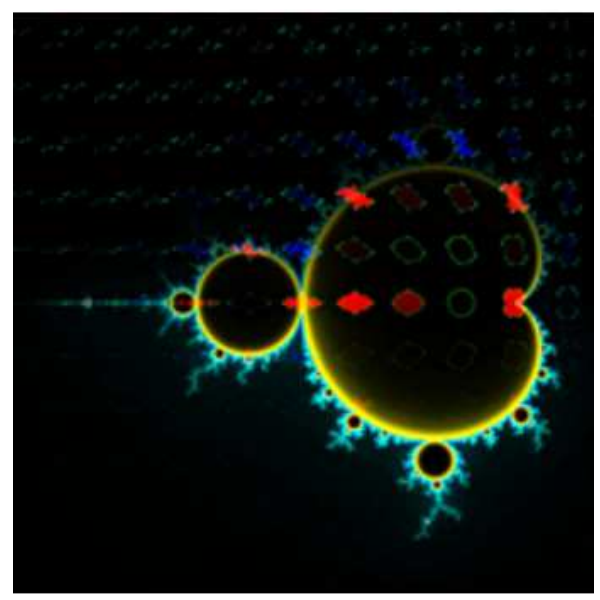

Figure 1. In black, image of the Mandelbrot Set superimposed with the filled Julia sets represented by some of its points (in red the connected Julia sets and in blue those not related). Available at: https://www.google.es/search? $q=$ image + of + the + Mandelbrot + Set + superimposed + with + the + filled + Julia + sets + represented\&tbm $=i s c h \&$ source $=i u \& i c t x=1 \&$ fir $=$ FjhFhTkh8iOZvM\%252CTzMDvC34brwOyM\%252C_\&vet=1\&usg=AI4_$k R H E t w 7 u b 7 Q d 2 A 2 A U 0 C S H 9 f o X B 7 w w \& s a=X \& v e d=2 a h U K E w i u z L y B 94 z t$ AhVJVK0KHWPjC88Q9QF6BAgJEAg\#imgrc $=$ FjhFhTkh8iOZvM Wikipedia, 2019.

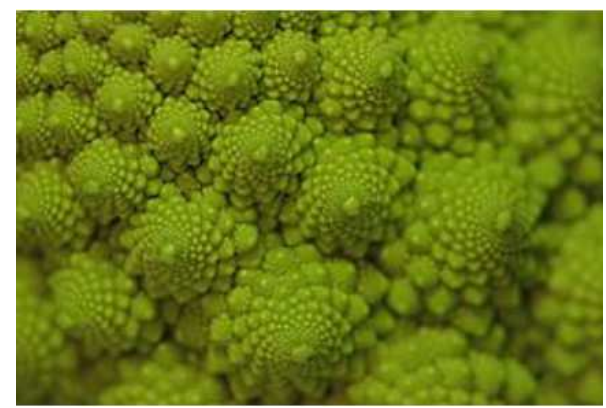

Figure 2. Natural fractals: are natural objects that can be represented with a very good approximation by mathematical fractals with statistical selfsimilarity. Fractals found in nature differ from mathematical fractals in that natural ones are approximate or statistical and their self-similarity extends only to a range of scales (for example, at a scale close to the atomic its structure differs from the macroscopic structure). Available at: https://www.google.es/search? $q=$ natural + fractals + images \&tbm $=i$ isch\&ved =2ahUKEwinivWw94ztAhUCO1MKHQGoCkIQ2-

$c C e g Q I A B A A \& o q=$ Natural + fractals\&gs_lcp $=C g N p b W c Q A R g B M g I I A D I E$ CAAQHjIECAAQHjIGCAAQBRAeMgYIABAIEB 46BAgAEBM6CAgAEAcQ HhATOgYIABAeEBM6CAgAEAUQHhATOgQIABBDOgUIABCXAzoECAAQ A1DKihRYvLwUYNPjFGgAcAB4AYABvAOIAcQUkgEJMC43LjAuMi4ymAE AoAEBqgELZ3dzLXdpeilpbWewAQDAAQE\&sclient $=i m g \& e i=C 4 S 1 X$ fYMYL2zAKBOKqQBA
This does not mean that the study and development of fractal mathematics is independent of material reality, on the contrary, its birth as a theory in the second half of the 20th century had as its fundamental objective, to model, describe and analyze many natural phenomena and scientific experiments that Euclidean or classical mathematics could neither represent nor answer.

Its visionary and conceptualizer Benoit Mandelbrot, a Polish mathematician nationalized in France and in the United States of America, a Jew who suffered the penalties of the Second World War, who was influenced by the work of mathematical geniuses such as Karl Theodor Wilhelm Weierstrass, Helge Von Koch, Waclaw Sierpinski, Henry Poincaré, Abram Samóilovich Bezikóvich, Pierre Fatou and Gaston Julia on the subject in question, proposed the term in 1975, which derives from the Latin fractus, which means broken or fractured.

Therefore, the following scientific problem is defined: How do we establish a mathematical model represented by radiological imagery?

The objective of this work is to confirm the behavior of the experimental data modeled by the fractal's dimension.

\section{Materials and Methods}

Theoretical methods are used: analysis - synthesis, induction - deduction, abstraction - concretion. The method to construct images, $[1,2]$ comprehension, explanation and interpretation processes. Mathematical methods, procedures and algorithms are applied.

Come true quest of information about the application of dimension fractal in the diagnostic one belonging to diseases, based in radiological imagery $[3,4]$.

Identify him correlated diseases the radiological imagery, the comparison becomes established between the histological and pathological elements, the confrontation is determined with the help of professional information-technology probranches $[5,6]$.

\section{Results}

In 1982, with his masterpiece Fractal Geometry of Nature, he exposed a whole universe, which, although not completely unknown, was loaded with a new and renewed vision of reality and the processes that develop it, and how the patterns by which Roughness and fractures are governed in nature and the chaotic behavior of many phenomena have an explanation and representation in the Fractal Dimension, not for pleasure he called this kind of mathematics the Fingerprint of God when they were reflected in everyday life.

The irregularity of the human body is its own characteristic. However, it has traditionally been measured with Euclidean metrics, by approximating its shapes to regular lines, areas and volumes. In response to this impossibility of making reliable measurements of this class of objects, fractal geometry is developed, which allows to adequately characterize the irregular shape of the human 
body. [1]

From fractal geometry, the fractal dimension is obtained, which is a numerical measure that represents the degree of irregularity of an object. [2] There is not a single fractal dimension, but rather a series of dimensions that are often equivalent but not always. There are therefore different ways to calculate the fractal dimension.

The abstract fractal is characterized mainly by symbisimilarity (similarity between the parts and the whole of the object), and is measured using the Hausdorff dimension. The natural fractal is characterized by the superposition between its parts and is studied by the boxcounting method, while in the statistical fractal, characterized by hyperbolic distributions, the Zipf / Mandelbrot law is applied.

The application of the fractal dimension within the field of medicine has been of great importance in clinical and experimental areas, in which objects such as neuronal and pulmonary structure, intestines and blood vessels of different structures, among others, have been characterized. [3] Regarding morphometric studies carried out in cancer, fractal geometry has been applied providing a more objective measure of clinical application $[4,5]$.

Specifically in the area of oncology, it has been possible to characterize cancer structures $[4,6,7]$, mammographic images, and to identify the appearance of primary cancer in cases of bone metastasis through the interpretation of images using the fractal dimension, to then compare data between normal and pathological morphological elements.

In summary, the objective that concerns us is the analysis of the use of this mathematical knowledge in the development of Medical Sciences, so we will present some investigations carried out since 2014 in a multidisciplinary way either by doctors, mathematicians and statisticians, where it will be reflected the importance and current need to use and develop these new quantitative mathematical analysis methods in favor of improving the quality of health services in our institutions, strengthening the veracity of clinical diagnoses for effective therapy and patient management.

In 2014 researchers from the Insight Group. Line of Deepening and Special Internship in Physics and Mathematics Applied to Medicine, Nueva Granada Military University. Center of Clinical Investigations of the Country in Bogotá, Colombia, a research project was carried out where a fractal diagnostic method was developed to evaluate cells of the cervix using the concept of Intrinsic Mathematical Harmony (AMI) and cell variability, which mathematically differentiates normal cells from cells.

L-SIL and H-SIL, making the diagnosis of ASCUS cells unnecessary, had the objective: to confirm the diagnostic capacity of the methodology developed by means of a blind study in comparison with the Gold Standard [8].

A new methodology was generated in which the fractal relationships of the preneoplastic and neoplastic cells of the squamous cells of the cervix are quantified, which were evaluated from the fractal dimension calculated for three cellular objects defined as: the nucleus, the cytoplasm without a nucleus and the entire cell. Likewise, the variability and Intrinsic Mathematical Harmony of these cells were measured.

Additionally, all cell prototypes representing each stage between normality and carcinoma were found, obtaining a generalization applicable to clinical practice. In this study, 18 prototypes of normality were found, while 44 prototypes of abnormality were found, including 8 of carcinoma [9].

The fractal dimension used to quantify the degree of irregularity of epithelial cells was the most widely used method for quantifying the irregularity of objects that have overlapping parts is the Box-Counting method. $[2,5,6]$

$$
\mathrm{D}=\frac{\log \mathrm{N}\left(2^{-(\mathrm{K}+1)}\right)-\log \mathrm{N}\left(2^{-K}\right)}{\log 2^{k+1}-\log 2^{k}}=\log _{2} \frac{\mathrm{N}\left(2^{-(\mathrm{K}+1)}\right)}{\mathrm{N}\left(2^{-K}\right)}
$$

In this expression $\mathrm{N}$ corresponds to the number of squares that the contour of the object contains, $\mathrm{K}$ corresponds to the degree of partition of the grid, and $\mathrm{D}$ represents the fractal dimension.

Cellular AMI (Intrinsic Mathematical Harmony) and Variability: Corresponds to the Degree of similarity or difference between the fractal dimensions of two of the defined objects, in this case the cytoplasm without nucleus and nucleus $(\mathrm{CN})$, the nucleus and the totality (NT) and cytoplasm without nucleus and totality (CT), obtained by finding the difference between the first decimal place of the fractal dimensions of the two objects to be compared.

Fractal cell prototype: corresponds to a simultaneous combination of numerical relationships between the fractal dimensions of the objects studied: nucleus $(\mathrm{N})$, cytoplasm without nucleus $(\mathrm{C})$ and totality $(\mathrm{T})$ evaluated with the AMI and Variability [8]

Fifty images of cells were selected from cervical-vaginal cytologies of women between 20 and 55 years of age, of which 5 have a cytological diagnosis within normal limits, 5 were diagnosed as ASCUS, 20 with L-SIL and 20 with HSIL. These images were obtained from the League Against Cancer, Bogotá Section.

They were observed at a magnification of $100 \mathrm{X}$ through a microscope, and then they were photographed with a digital camera to finally transfer them through a serial interface to the computer.

The photographs acquired dimensions of $1200 \times 1600$ pixels, and were viewed at $50 \%$ on the computer for analysis. Three mathematical objects to be measured were defined, based on previous studies (21): nucleus $(\mathrm{N})$, cytoplasm without nucleus (C) and totality (T) of each cell, calculating their fractal dimension with the Box-Counting method from five grids made up of squares with $2,4,8,16$ and $32 \mathrm{~mm}$ sides [8]

On the images, the fractal dimension was calculated with the simplified Box-Counting method for the three defined mathematical objects, using previously developed $\mathrm{C}++$ language software. Subsequently, their measurements of cellular AMI and Variability were established for the three comparisons obtained: $\mathrm{C}$ and $\mathrm{N}, \mathrm{N}$ and $\mathrm{T}$ and $\mathrm{C}$ and $\mathrm{T}$. 
As a result, the fractal dimensions of the cytoplasm of the normal cells measured were between 0.1805 and 1.9958; for the nucleus, it was found that the fractal dimensions were between 0.2462 and 1.986 and for the whole; values between 0.1968 and 1.9805 were obtained. The variability and AMI values of the comparison between Cytoplasm and Nucleus were found between 0 and 5, as well as between Nucleus and Totality, while for the comparison between Cytoplasm and Totality, values of $0-1$ were obtained [8].

It was found that the 10 samples diagnosed as normal from the conventional parameters were diagnosed as normal based on the physical-mathematical methodology, presenting AMI values and variability of $\mathrm{C}$ and $\mathrm{N}$, and of $\mathrm{N}$ and $\mathrm{T}$ between 3 and 5 in all cases, as well as values between 0 and 1 in the comparison $\mathrm{C}$ and $\mathrm{T}$. The $20 \mathrm{~L}-\mathrm{SIL}$ and the $20 \mathrm{H}-\mathrm{SIL}$ also presented the same mathematical and histological diagnosis; the former presented values between 0 and 2 for the three comparisons in all cases, while the latter presented values of 0 for the three comparisons in all cases.

\section{Discussion}

Cellular AMI (Intrinsic Mathematical Harmony) and Variability: Corresponds to the Degree of similarity or difference between the fractal dimensions of two of the defined objects, in this case the cytoplasm without nucleus and nucleus $(\mathrm{CN})$, the nucleus and the totality (NT) and cytoplasm without nucleus and totality (CT), obtained by finding the difference between the first decimal place of the fractal dimensions of the two objects to be compared.

Fractal cell prototype: corresponds to a simultaneous combination of numerical relationships between the fractal dimensions of the objects studied: nucleus $(\mathrm{N})$, cytoplasm without nucleus (C) and totality (T) evaluated with the AMI and Variability [8].

Fifty images of cells were selected from cervical-vaginal cytologies of women between 20 and 55 years of age, of which 5 have a cytological diagnosis within normal limits, 5 were diagnosed as ASCUS, 20 with L-SIL and 20 with HSIL. These images were obtained from the League Against Cancer, Bogotá Section.

They were observed at a magnification of $100 \mathrm{X}$ through a microscope, and then they were photographed with a digital camera to finally transfer them through a serial interface to the computer.

The photographs acquired dimensions of $1200 \times 1600$ pixels, and were viewed at $50 \%$ on the computer for analysis. Three mathematical objects to be measured were defined, based on previous studies (21): nucleus $(\mathrm{N})$, cytoplasm without nucleus $(\mathrm{C})$ and totality $(\mathrm{T})$ of each cell, calculating their fractal dimension with the Box-Counting method from five grids made up of squares with 2, 4, 8, 16 and $32 \mathrm{~mm}$ sides [8].

On the images, the fractal dimension was calculated with the simplified Box-Counting method for the three defined mathematical objects, using previously developed $\mathrm{C}++$ language software. Subsequently, their measurements of cellular AMI and Variability were established for the three comparisons obtained: $\mathrm{C}$ and $\mathrm{N}, \mathrm{N}$ and $\mathrm{T}$ and $\mathrm{C}$ and $\mathrm{T}$.

As a result, the fractal dimensions of the cytoplasm of the normal cells measured were between 0.1805 and 1.9958; for the nucleus, it was found that the fractal dimensions were between 0.2462 and 1.986 and for the whole; values between 0.1968 and 1.9805 were obtained. The variability and AMI values of the comparison between Cytoplasm and Nucleus were found between 0 and 5 , as well as between Nucleus and Totality, while for the comparison between Cytoplasm and Totality, values of $0-1$ were obtained [8].

It was found that the 10 samples diagnosed as normal from the conventional parameters were diagnosed as normal based on the physical-mathematical methodology, presenting AMI values and variability of $\mathrm{C}$ and $\mathrm{N}$, and of $\mathrm{N}$ and $\mathrm{T}$ between 3 and 5 in all cases, as well as values between 0 and 1 in the comparison $\mathrm{C}$ and T. The $20 \mathrm{~L}-\mathrm{SIL}$ and the $20 \mathrm{H}-\mathrm{SIL}$ also presented the same mathematical and histological diagnosis; the former presented values between 0 and 2 for the three comparisons in all cases, while the latter presented values of 0 for the three comparisons in all cases.

It was additionally found that of the five ASCUS cells evaluated, one of them presented values corresponding to normality, three to L-SIL and one to H-SIL, confirming that this stage does not present mathematical characteristics that allow it to be differentiated from the others, and that therefore On the contrary, it is possible to clarify its diagnosis through this procedure, establishing whether its mathematical measurements correspond to one or another stage.

Table 1. Fractal dimension measures fractal variability and cellular AMI, including normal, ASCUS, L-SIL and H-SIL. C: Cytoplasm; N: Core; T: Totality. Dx: Mathematical opinion.

\begin{tabular}{|c|c|c|c|c|c|c|c|}
\hline \multirow[t]{2}{*}{ No. } & \multirow{2}{*}{$\mathbf{D}_{\mathbf{x}}$} & \multicolumn{3}{|c|}{ Fractal Dimension } & \multicolumn{3}{|c|}{$\begin{array}{l}\text { Fractal variability and } \\
\text { cellular AMI }\end{array}$} \\
\hline & & $\mathbf{C}$ & $\mathbf{N}$ & $\mathbf{T}$ & $\mathbf{C N}$ & $\mathbf{N} \mathbf{T}$ & $\mathbf{C} \mathbf{T}$ \\
\hline 1 & H-SIL & 1.5809 & 1.5328 & 1.5379 & 0 & 0 & 0 \\
\hline 2 & H-SIL & 1.6891 & 1.6469 & 1.6596 & 0 & 0 & 0 \\
\hline 3 & H-SIL & 0.9382 & 0.9986 & 0.9521 & 0 & 0 & 0 \\
\hline 4 & H-SIL & 0.7386 & 0.7819 & 0.7168 & 0 & 0 & 0 \\
\hline 5 & H-SIL & 1.6162 & 1.6784 & 1.6309 & 0 & 0 & 0 \\
\hline 6 & H-SIL & 1.5347 & 1.5169 & 1.5831 & 0 & 0 & 0 \\
\hline 7 & H-SIL & 0.2903 & 0.2718 & 0.212 & 0 & 0 & 0 \\
\hline 8 & H-SIL & 0.2002 & 0.2462 & 0.2626 & 0 & 0 & 0 \\
\hline 9 & H-SIL & 1.9561 & 1.986 & 1.9457 & 0 & 0 & 0 \\
\hline 10 & H-SIL & 1.153 & 1.1821 & 1.9457 & 0 & 0 & 0 \\
\hline 11 & L-SIL & 0.8489 & 0.6414 & 0.7837 & 2 & 1 & 1 \\
\hline 12 & L-SIL & 1.3565 & 1.5471 & 1.6384 & 1 & 1 & 0 \\
\hline 13 & L-SIL & 1.8618 & 1.6366 & 1.7702 & 2 & 1 & 1 \\
\hline 14 & L-SIL & 1.4479 & 1.5471 & 1.4491 & 1 & 1 & 0 \\
\hline 15 & L-SIL & 0.2488 & 0.5449 & 0.3693 & 2 & 1 & 1 \\
\hline 16 & L-SIL & 0.6945 & 0.6819 & 0.7923 & 0 & 1 & 1 \\
\hline 17 & L-SIL & 0.4104 & 0.6441 & 0.5746 & 2 & 1 & 1 \\
\hline 18 & L-SIL & 1.5053 & 1.6668 & 1.4647 & 1 & 2 & 1 \\
\hline 19 & L-SIL & 0.1805 & 0.3499 & 0.1968 & 2 & 2 & 0 \\
\hline 20 & Normal & 1.7576 & 1.5131 & 1.7463 & 3 & 3 & 0 \\
\hline 21 & Normal & 1.6204 & 1.9378 & 1.6716 & 3 & 3 & 0 \\
\hline 22 & Normal & 1.6855 & 1.9378 & 1.6716 & 3 & 3 & 0 \\
\hline 23 & Normal & 1.9968 & 1.4836 & 1.9805 & 5 & 5 & 0 \\
\hline 24 & Normal & 1.4949 & 1.9211 & 1.5202 & 5 & 4 & 1 \\
\hline 25 & Normal & 1.1258 & 1.532 & 1.1783 & 4 & 4 & 0 \\
\hline
\end{tabular}


Fractal dimension measures fractal variability and cellular AMI of 25 of the evaluated samples, including 4 normal, 5 ASCUS, 7 L-SIL and 9 H-SIL. C: Cytoplasm; N: Core; T: Totality. Dx: Mathematical opinion, which is the same conventional diagnosis in all cases except for the ASCUS cells, which correspond to the shaded rows, showing that this type of cells can present mathematical behaviors analogous to normality, L-SIL or H-SIL [8].

As a final result, a comparison was established between the conventional and the physical-mathematical diagnosis, in order to demonstrate the degree of certainty that the methodology has to characterize the degrees of alteration ranging from normality to carcinoma, showing that through this procedure it is It is possible to mathematically diagnose normal cells, L-SIL and H-SIL with $100 \%$ sensitivity and specificity.

Another work where the use of the fractal dimension is used through box-counting is in Mathematical characterization of Legg-Calvé-Perthes disease through fractal geometry, carried out by a group of scientists from the Nueva Granada Military University.

In which they developed a new methodology for the mathematical, objective and reproducible characterization of the diagnostic Perthes radiography, from the frontal radiographic images obtained from the hip joint, using fractal geometry.

Methods: descriptive exploratory study in which the morphology of the femur head was evaluated through the fractal dimension obtained by the Box-Counting method, applied to 20 radiographic plates with Legg-Calve-Perthes disease, establishing a new objective morphometric measurement for this disease $[9,10]$.

Table 2. The fractal dimensions of the defined objects ranged. The number of occupied squares varied, and grids respectively.

\begin{tabular}{|c|c|c|c|c|c|c|}
\hline \multirow{2}{*}{ Patients } & \multicolumn{5}{|c|}{ Ocupation values } & \multirow{2}{*}{$\begin{array}{l}\text { Fractal } \\
\text { Dimension }\end{array}$} \\
\hline & 2 & 4 & 8 & 16 & 32 & \\
\hline 1 & 543 & 277 & 144 & 75 & 39 & 0.937545 \\
\hline 2 & 593 & 318 & 168 & 84 & 41 & 0.962924 \\
\hline 3 & 595 & 322 & 167 & 87 & 47 & 0.921229 \\
\hline 4 & 611 & 325 & 171 & 91 & 49 & 0.940524 \\
\hline 5 & 431 & 228 & 118 & 61 & 32 & 0.956023 \\
\hline 6 & 388 & 202 & 108 & 53 & 27 & 0.949292 \\
\hline 7 & 573 & 314 & 163 & 81 & 42 & 0.947113 \\
\hline 8 & 612 & 330 & 172 & 86 & 45 & 0.96909 \\
\hline 9 & 539 & 296 & 152 & 76 & 37 & 0.945657 \\
\hline 10 & 586 & 314 & 162 & 83 & 43 & 0.978928 \\
\hline 11 & 475 & 256 & 129 & 63 & 32 & 0.950538 \\
\hline 12 & 479 & 249 & 132 & 68 & 34 & 0.947484 \\
\hline 13 & 466 & 250 & 129 & 66 & 34 & 0.924044 \\
\hline 14 & 463 & 245 & 126 & 67 & 36 & 0.94061 \\
\hline 15 & 526 & 276 & 144 & 74 & 39 & 0.898152 \\
\hline 16 & 549 & 291 & 156 & 82 & 46 & 0.94243 \\
\hline 17 & 546 & 295 & 153 & 80 & 40 & 0.957292 \\
\hline 18 & 467 & 251 & 129 & 66 & 33 & 0.945688 \\
\hline 19 & 587 & 313 & 161 & 83 & 43 & 0.945197 \\
\hline 20 & 577 & 308 & 162 & 83 & 42 & 0.937545 \\
\hline
\end{tabular}

The fractal dimensions of the defined objects ranged from 0.978928 to 0.898152 . The number of occupied squares varied between 612 and 388, and between 49 and 27 for the 2 and $32 \mathrm{~cm}$ grids respectively (table). The feasibility of obtaining objective morphometric-type measurements from radiological images for the evaluation of Legg-Calvé-Perthes disease is then shown (table) from the fractal theory.

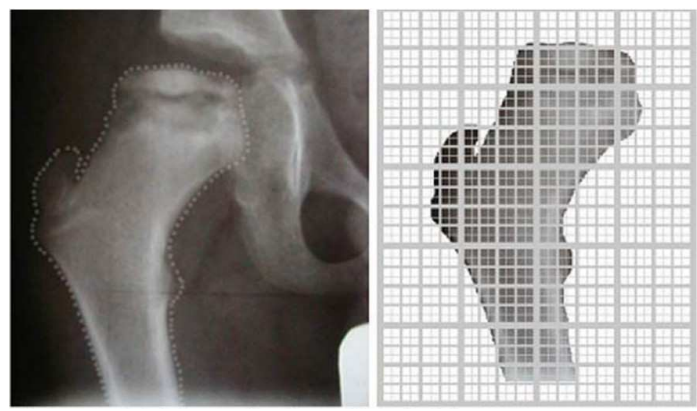

Figure 3. Pleasing [10]

Occupation in each of the grids of the measured radiographic images and corresponding fractal dimension.

There are other studies that use the fractal dimension for the analysis of radiological images and pathological histological samples, as an alternative diagnostic method to conventional methods. [11-14]

The need for a new technology is permissible to satisfy diagnostic methods in an age full of scientific events. The use of fractal mathematics in response to this need in the field of medical sciences, overcoming the barriers of traditional tools, it is necessary, of course. [15-18]

That much more research is needed in this field to perfect its use and of course that it is demanded by a universalization of the subject in question. More than proven in the veracity of the data obtained from the calculation of fractal dimensions and its use in the diagnosis and evaluation of some diseases, the mathematics is exact, there is no margin for errors of enormous magnitude, when the operations.

It is the part of this science that current medicine needs, timely accuracy that means greater prolongation of life and less suffering for our patients and families.

\section{Conclusions}

A methodology of work based in radiological imagery by comparison of histological and pathological elements to determine different diseases in patients becomes established.

An approximation fractal can be useful like one of processing's methods to interpret the most courteous details of the medical imagery and the physiological signs. However, a lot more experience to evaluate his diagnostic or predictive value for the patient's care is needed.

There is still a long way to go, but the important thing is to be on the right track, leave the fear of the unknown and embrace the new ways of doing medicine.

\section{References}

[1] Vasiljevic J, Reljin B, Sopta J, Mijucic V, Tulic G, Reljin I. Application of multifractal analysis on microscopic images in the classification of metastatic bone disease. Biomed Micro devices. 2012; 14: 541-548. 
[2] Abedini M, Bowling A, Chakravorty R, Demyanov S, Garnavi R. Detection of outlier lesions based on extracted features from skin images, 2018. US Patent US 2018/0122065 A1.

[3] Hermann P; Mrkvirka T; et al. Fractal; stochastic geometry inference for breast cancer: a case study with random fractal models; Quermass-interaction process, Statistics in Medicine, vol. 34. 2015. https://doi.org/10.1002/sim.6497

[4] Pribic J; Kanjer K; et al. Fractal dimension; lacunarity of tumor microscopic images as prognostic indicators of clinical outcome in early breast cancer. Biomarkers in Medicine, vol. 9, pag. 1279-1290. 2015. https://doi.org/10.2217/bmm.15.102

[5] Shanmugavadivu P; Sivakumar V; et al. Fractal dimensionbound spatiotemporal analysis of digital mammograms. The European Physical Journal Special Topics, vol. 225, 2016, pag. $137-146$.

[6] Fiz J. New diagnostic techniques for respiratory diseases. Image Analysis. March 17. 2016. Hospital Universitario Germans Trias I Pujol. Servicio de Neumología. Spain.

[7] Luzi P, Bianciardi G, Miracco C, Desanti MM, Del Vecchio MT, Alia L, et al. Fractal analysis in human pathology. Ann NY Acad Sci. 1999; 879: 255-57.

[8] Gazit Y, Baish JW, Safabaksh N. Fractal characteristics of tumor vascular architecture during tumor growth and regression. Microcirculation. 1997; 4: 395-402.

[9] Baish H, Jain R. Fractals and Cancer. Cancer research. 2000; 60: 3683-3688.

[10] Sankar D, Thomas T. A New Fast Fractal Modeling Approach for the Detection of Microcalcifications in Mammograms. Journal of Digital Imaging. 2010; 23 (5): 538-546.
[11] Rodríguez J. Fractales: Ayuda diagnóstica para células preneoplásicas y cancerígenas del epitelio escamoso cervical confirmación de aplicabilidad clínica. Revista 24 (1): 79-88, 2016.

[12] Rodríguez J, Prieto S, Correa C, Posso H, Bernal P, ViteryS, et al. Generalización fractal de células preneoplásicas y cancerígenas del epitelio escamoso cervical de aplicaciónclínica. Rev Med. 2010; 18 (2): 173-181.

[13] West J. Fractal physiology and chaos in medicine. Singapore: World Scientific; 1990.

[14] Dobrescu R; Ichim L; et al. Benignand malignant breast tumors: Diagnosis using fractal measures. In 2014 18th International Conference on System Theory, Control; Computing, ICSTCC 2014, Faculty of Automatic Control; Computers, Politehnica University of Bucharest, pag. 82-86.

[15] Ascencio A, Zapata J. Nevus classification by calculating the fractal dimension and the harmonic analysis of the contour extracted from multispectral images. Revista INGENIERÍA UC, vol. 25, núm. 2, 2018 Universidad de Carabobo.

[16] Liang Y, Sun L, Ser W, Lin F, Tien S, Chen O, Lin Z. Classification of non-tumorous skin pigmentation disorders using voting based probabilistic linear discriminant analysis. Computers in Biology and Medicine, 2018.

[17] Fernandez S, Rngel F. Comparativo de los Algoritmos de Dimensión Fractal Higuchi, Katz y Multiresolución de Conteo de Cajas en Señales EEG Basadas en Potenciales Relacionados por Eventos. September 2017. Revista EIA 14 (27): 7317.

[18] Mandelbrot B. Introducción. En: Mandelbrot B. Los Objetos Fractales. Barcelona. Tusquets Eds. S. A.; 2000. p. 13-26. US 2018/0122065 A1. 